\title{
Computationally Efficient, Fully Coupled Multiscale Modeling of Materials Phenomena Using Calibrated Localization Linkages
}

\author{
Surya R. Kalidindi \\ Department of Mechanical Engineering and Mechanics, Drexel University, Philadelphia, PA 19104, USA \\ Correspondence should be addressed to Surya R. Kalidindi, skalidin@coe.drexel.edu
}

Received 26 August 2012; Accepted 17 October 2012

Academic Editors: F. M. Labajos and T. Xu

Copyright () 2012 Surya R. Kalidindi. This is an open access article distributed under the Creative Commons Attribution License, which permits unrestricted use, distribution, and reproduction in any medium, provided the original work is properly cited.

\begin{abstract}
Most modern physics-based multiscale materials modeling and simulation tools aim to take into account the important details of the material internal structure at multiple length scales. However, they are extremely computationally expensive. In recent years, a novel data science enabled framework has been formulated for effective scale-bridging that is central to practical multiscaling. A salient feature of this new approach is its ability to capture heterogeneity of fields of interest at different length scales. In this approach, the computations at the mesoscale are handled using a novel data science approach called materials knowledge systems (MKS). The MKS approach has enjoyed tremendous success in building highly accurate and computationally efficient metamodels for localization (i.e., mesoscale spatial distribution of a macroscale imposed field such as stress or strain rate) in simulating a number of different multiscale materials phenomena. MKS derives its accuracy from the fact that it is calibrated to results from previously established numerical models for the phenomena of interest, while its computational efficiency comes from the use of fast Fourier transforms. The current capabilities and the future outlook for the MKS framework are expounded in this paper.
\end{abstract}

\section{Introduction}

Materials with enhanced performance characteristics have served as critical enablers for the successful development of advanced technologies throughout human history and have contributed immensely to the prosperity and well-being of various nations. It is important to recognize that the multifunctional performance characteristics of a material are not controlled by its chemical composition alone, but by the myriad details of its hierarchical three-dimensional (3D) internal structure that spans a very broad range of length scales (from the electronic to the macroscale) and evolves over a broad range of time scales. Although the fundamental connections between the material's internal structure, its evolution through various manufacturing processes, and its macroscale properties (or performance characteristics) in service are known to exist and are fully acknowledged, standard protocols (i.e., broadly adopted workflows) for establishing this knowledge base do not yet exist. The traditional approaches of materials development have relied heavily on experimentation and are known to be expensive and slow (typically takes about 20 years to introduce a new material in critical aerospace applications [1-6]). It is evident that the time and resource consumptive empiricism that has dominated materials development during the past century must give way to a greater dependence on modeling and simulation [2] and modern data science tools [3-5]. Two recent reports from the National Science and Technology Council (NTSC) $[3,6]$ (a cabinet-level council within the Executive Office of the President of United States of America) have emphasized the critical importance of high-performance materials to our nation's economy, prosperity, and security. These latest strategic initiatives (Materials Genome Initiative for Global Competitiveness, 2011; A National Strategic Plan for Advanced Manufacturing, 2012) call for the creation of a new materials innovation infrastructure to facilitate the design, manufacture, and deployment of new high-performance materials at a dramatically accelerated pace in emerging advanced technologies.

Success in the endeavors described above critically hinges on the availability of high-fidelity modeling and simulation tools for various materials phenomena controlling the performance characteristics of interest in advanced technologies. The demand for high fidelity requires a physics-based 
multiscale approach that captures the salient aspects of the inherent heterogeneity at various constituent scales and communicates them accurately to both the higher as well as the lower scales. This is indeed a highly challenging task. A 2006 NSF Report [7] has identified "The Tyranny of Scales: The Challenge of Multiscale Modeling and Simulation" as one of the core challenges for advances in simulation-based engineering science (SBES). This report suggests that the conventional multiscale modeling approaches are incapable of addressing physical phenomena operating across the large range of length scales encountered in the design of advanced materials and that fundamentally new concepts and approaches are essential to address this challenge. The critical need for new transformative approaches for multiscale modeling (both spatial and temporal) was also emphasized in a more recent report from the Committee on Integrated Computational Materials Engineering (ICME) of the National Materials Advisory Board (NMAB) [5].

It is well known that the knowledge of the chemical composition of a material alone is insufficient to establish practical bounds on many of its physical properties of interest for applications in advanced technologies [8-19]. The threedimensional details of the material internal structure (hereafter generically referred to as the material microstructure) are often richly complex and span a multitude of length scales [20-36]. These details play a pivotal role in controlling the material's overall multifunctional performance characteristics. For example, most materials being explored for structural applications are metals (e.g., Ti alloys in jet engines [3754], advanced high-strength steels [55-66] and $\mathrm{Mg}$ alloys [67-83] in lightweight automobiles, and $\mathrm{Zr}$ alloys in nuclear industry [84-88]), whose internal structures at the mesoscale exhibit multiphase polycrystalline topologies with potentially complex heterogeneous distribution of various defects (e.g., pores, distribution of cracks, and dislocation density distributions). A major challenge for multiscale materials modeling is the rigorous quantification of the material microstructure that adequately captures all of its salient features-at least those features that demonstrate a significant role in the performance characteristics of interest $[4,9$, 19, 89-97].

Given the broad range of length scales involved in quantifying the material microstructure, it becomes essential to invoke the concept of a representative volume element (RVE) at each length scale to facilitate multiscale modeling and simulation of materials phenomena. Furthermore, it is often unavoidable to invoke the assumption of statistical homogeneity, that is, any (sufficiently large) RVE chosen from a material sample will exhibit the same structure in a statistical sense. Consequently, one needs to define the RVE with sufficient rigor. Although a single definition of an RVE has not yet been broadly adopted by the materials community, the generally employed concepts of an RVE implicitly identify a finite region that is randomly sampled from an infinite medium and is "statistically representative" of the whole, while also serving as a suitable basis for effectively smoothing out the local spatial heterogeneities in such a way that the macroscale properties of the sample are captured to a desired accuracy via homogenization theories [18, 98-106].
Although not explicitly stated, it is generally implied that the RVE replicates adequately not only the macroscale anisotropic properties and performance of the much larger sample, but also the salient statistical measures of the microstructure (e.g., $n$-point statistics of the microstructure $[9,44$, $89,107-110]$ ). Refinements to the concept of RVEs have been provided in recent literature [44, 109-112].

This brings us to the central challenge of multiscale modeling. How does one communicate relevant information between the constituent length (and time) scales? Broadly speaking, there have been two main approaches in the literature to address this challenge. In the first approach, the spatial domain is refined to the desired length scale only in selected regions (presumably regions where there is a high concentration of the fields of interest, e.g., stress or strain in mechanical problems), and suitable matching conditions are imposed at the boundaries where they meet the material that is spatially refined only at the higher length scale (e.g., [113117]). Needless to say, this approach encounters significant computational difficulty as it is fairly difficult to satisfy the imposed matching boundary conditions, especially at the interfaces between the regions that are spatially refined at different length scales. In a second approach referred to as hierarchical multiscale modeling (e.g., $[9,118-121]$ ), relevant information between the constituent length scales is exchanged in a statistical framework using the concept of the RVE described earlier. Although both these approaches enjoy several potential advantages relative to each other in different multiscale problems, it is clear that hierarchical modeling is much more practical and better suited for considerations of multiscale materials design [5]. Not surprisingly, hierarchical modeling has been the method of choice in almost all multiscale materials design efforts to date $[63-65,119,121-$ 124].

The main drawback of current hierarchical modeling approaches is that they usually employ various simplifying assumptions and, therefore, trade accuracy for computational speed. On the other hand, the scale-bridging relationships used in hierarchical modeling are computationally better suited for capturing quantitatively the specific influence of any selected microstructure parameter on the macroscale material response of interest. This information is central to the materials design efforts $[9,118,119,121]$. In most hierarchical modeling approaches to date, the focus has been in communicating the effective properties to the higher length scales, that is, on homogenization. There is often very little information passed in the opposite direction, that is, localization. As an example, localization might involve the spatial distribution of the response field of interest (e.g., stress or strain rate fields) at the microscale for an imposed loading condition at the macroscale. For fully coupled multiscale simulations, localization has to be accomplished by satisfying the governing field equations at the lower length scales. In several materials design problems (e.g., in simulating thermomechanical processes on materials where there is macroscale heterogeneity in the evolution of the underlying microstructure), localization is just as important as homogenization, if not more important. Furthermore, if 
localization is addressed with adequate accuracy, it implicitly results in much higher accuracy for homogenization.

The fidelity of the homogenization and localization relationships used in hierarchical multiscale modeling can be significantly improved by adopting modern data science approaches. This is the central idea behind the recently formulated scale-bridging framework called microstructure knowledge systems (MKS) [13, 125-128]. Building on the statistical continuum theories developed by Kroner [129, 130], MKS establishes high-fidelity microstructure propertyprocessing relationships that are amenable for bidirectional exchange of information between the constituent hierarchical length scales. In the MKS framework, the localization relationships of interest are expressed as calibrated metamodels that take the form of a simple algebraic series whose terms capture the individual contributions from a hierarchy of local microstructure descriptors. Each term in this series expansion is expressed as a convolution of the appropriate local microstructure descriptor and its corresponding local influence at the microscale. The series expansion in the MKS framework is in complete accord with the series expansion obtained in the statistical continuum theories developed by Kroner $[129,130]$. However, the MKS approach dramatically improves the accuracy of these expressions by calibrating the convolution kernels in these expressions to results from previously validated physics-based models. In recent work [126], the MKS approach was demonstrated to successfully capture the tails of the microscale stress and strain distributions in composite systems with relatively high contrast, using the higher-order terms in the localization relationships. It was also demonstrated that the MKS approach can be applied to problems involving nonlinear material behaviour such as spinodal decomposition [125] and rigid plastic deformation [127].

\section{Materials Knowledge Systems Framework}

The MKS framework is derived from generalized composite theories for effective response of heterogeneous materials $[18,19,93,129,130]$. Inherent to these theories is the concept of a scale-bridging localization tensor that relates the local fields of interest at the microscale to the macroscale (typically averaged) fields. For example, the fourth-rank localization tensor for elastic deformation of a composite material, a, relates the local elastic strain at any location of interest in the microstructure, $\boldsymbol{\varepsilon}(\mathbf{x})$, to the macroscale strain imposed on the composite, $\langle\boldsymbol{\varepsilon}(\mathbf{x})\rangle$, as

$$
\begin{gathered}
\boldsymbol{\varepsilon}(\mathbf{x})=\mathbf{a}(\mathbf{x})\langle\boldsymbol{\varepsilon}(\mathbf{x})\rangle, \\
\mathbf{a}(\mathbf{x})=\left(\mathbf{I}-\left\langle\boldsymbol{\Gamma}^{\mathbf{r}}\left(\mathbf{x}, \mathbf{x}^{\prime}\right) \mathbf{C}^{\prime}\left(\mathbf{x}^{\prime}\right)\right\rangle\right. \\
\left.+\left\langle\boldsymbol{\Gamma}^{\mathbf{r}}\left(\mathbf{x}, \mathbf{x}^{\prime}\right) \mathbf{C}^{\prime}\left(\mathbf{x}^{\prime}\right) \mathbf{\Gamma}^{\mathrm{r}}\left(\mathbf{x}^{\prime}, \mathbf{x}^{\prime \prime}\right) \mathbf{C}^{\prime}\left(\mathbf{x}^{\prime \prime}\right)\right\rangle-\cdots\right) .
\end{gathered}
$$

In (1) and (2), I is the fourth-rank identity tensor, $\mathbf{C}^{\prime}(\mathbf{x})$ is the deviation in the local elastic stiffness at spatial location $\mathbf{x}$ with respect to that of a selected reference medium, $\Gamma^{\mathbf{r}}$ is a symmetrized derivative of the Green's function defined using the elastic properties of the selected reference media, and $\langle\cdots\rangle$ brackets denote ensemble averages over representative volume elements (RVEs). It should be noted that (1) and (2) automatically satisfy the governing equilibrium equations at the mesoscale $[129,130]$; truncation of the series in (2), however, introduces errors.

The evaluation of the terms in the series expression in (2) requires knowledge of higher-order spatial correlations of local states in the microstructure (related to the $n$-point statistics of the microstructure $[89,107,108])$. Local states are typically identified by a combination of distinguishable microstructural parameters of interest (such as phase, lattice orientation, and composition) that can be quantified (or at least labeled). The first $\langle\cdots\rangle$ term on the right-hand side in (2) captures the contribution to the tensor $\mathbf{a}(\mathbf{x})$ from the local state at point $\mathbf{x}^{\prime}$ in the material. In a very similar manner, the second $\langle\cdots\rangle$ term in (2) reflects the contribution from two local states at points $\mathbf{x}^{\prime}$ and $\mathbf{x}^{\prime \prime}$ to $\mathbf{a}(\mathbf{x})$.

There exist two main difficulties in the computation of the localization tensor defined in (2). The first difficulty stems from the evaluation of the ensemble averages that are in fact convolution integrals whose integrands exhibit singularities (also known as the principal value problem). The second difficulty is that the accuracy of the solutions obtained is quite sensitive to the selection of the reference medium [131]. It should also be noted that the expression of the localization tensor in (2) does not lend itself to a scheme where some of the calculations performed for one microstructure may be efficiently carried forward to the calculations for a different microstructure. In other words, any changes in the microstructure would force one to reevaluate almost all of the terms in the series expansion.

The convolution expressions in (2) can be conveniently cast into discrete Fourier transform (DFT) space [132, 133] to allow exploitation of the fast Fourier transform (FFT) algorithms in seeking solutions to the localization relationship (also called Lippmann-Schwinger equation). Indeed, this approach has been explored by many authors in the literature (e.g., [14, 134-137]). However, these approaches continue to require relatively high computational resources because of the iterative schemes employed in the solution methodologies. Although, the approaches described in prior literature are very useful for capturing the intricate details of the microscale response in a single RVE, they are not well suited for addressing inverse problems in materials design (where a very large number of microstructures need to be evaluated and screened) or for conducting practical multiscale simulations where every material point at the macroscopic level is to be associated with a representative threedimensional microstructure. In recent papers [13, 125-128], we presented a new mathematical framework to cast (2) into a computationally efficient, potentially invertible, scalebridging linkage that is especially suited for multiscale design and analyses of composite microstructures. A central element of this new framework is the transformation of (2) into an efficient spectral (Fourier) form that decouples the terms capturing the physics (called influence functions) from the terms capturing the microstructure topology (called microstructure function). 
In the MKS framework, we utilize the concept of a microstructure function [90]. In this description, the threedimensional spatial domain of the material internal structure is discretized into a uniform grid of spatial cells (or voxels) indexed by $\mathbf{s} \in \mathbf{S}$. Let $|\mathbf{S}|$ denotes the number of spatial cells in the tessellated microstructure domain. The microstructure datasets identify the amount of each local state (identified by selected combination of phase identifiers, elemental compositions, crystal lattice orientations, etc.) present in each spatial cell. The set of all distinct local states that are possible in a given material system is referred to as the local state space, which in many cases is multidimensional because the local state is often represented by a set of multiple parameters. The multidimensional local state space is tessellated into individual bins enumerated by $h=$ $1,2, \ldots, H$. Following the approach proposed by Adams et al. [90], the digital microstructure function denoted by $m_{\mathrm{s}}^{h}$ is defined as the volume fraction in the spatial cell $s$ occupied by all local states in bin $h$. Based on this definition of a digital microstructure variable, it is easy to establish the following properties:

$$
\sum_{h=1}^{H} m_{\mathrm{s}}^{h}=1, \quad 0 \leq m_{\mathrm{s}}^{h} \leq 1, \quad \frac{1}{|\mathbf{S}|} \sum_{\mathrm{s} \in \mathbf{S}} m_{\mathrm{s}}^{h}=V^{h},
$$

where $V^{h}$ denotes the volume fraction of local state $h$ in the entire microstructure dataset.

Note that the framework for the description of the microstructure presented above is fairly general and can accommodate any combination of material features by simply increasing the dimensionality of the local state space; it is also not limited to any specific length or time scales. The discretized representation of microstructure offers many advantages in fast computation of microstructure measures/metrics [9, 89], automated identification of salient microstructure features in large datasets [138], extraction of representative volume elements from an ensemble of datasets $[110,111]$, reconstructions of microstructures from measured statistics $[139,140]$, building of real-time searchable microstructure databases [44], and mining of high-fidelity multiscale structure-performance-structure evolution linkages from physics-based models (the main focus of this paper) $[13,125-128]$.

Let $\langle\mathbf{p}\rangle$ denote the macroscale imposed variable (e.g., local stress, strain, or strain rate tensors) that needs to be spatially distributed in the microstructure as $\mathbf{p}_{\mathbf{s}}$ for each spatial cell indexed by $\mathbf{s}$. In all of the example case studies completed thus far in the MKS framework, the physical quantities of interest were chosen such that $\langle\mathbf{p}\rangle$ is indeed equal to the volume averaged value of $\mathbf{p}_{\mathrm{s}}$ over the microscale. In the MKS framework, the localization relationship, extended from Kroner's statistical continuum theories $[129,130]$, captures the local response field in the microstructure using a set of kernels and their convolution with higher-order descriptions of the local microstructure (i.e., local neighborhood).
The localization relationship can be expressed as a series sum $[13,125-128]$ :

$$
\begin{aligned}
\mathbf{p}_{s}= & \left(\sum_{h=1}^{H} \sum_{\mathbf{t} \in \mathbf{S}} \boldsymbol{\alpha}_{\mathbf{t}}^{h} m_{\mathbf{s}+\mathbf{t}}^{h}+\sum_{h=1}^{H} \sum_{h^{\prime}=1}^{H} \sum_{\mathbf{t} \in \mathbf{S}}^{S} \sum_{\mathbf{t}^{\prime} \in \mathbf{S}}^{S} \boldsymbol{\alpha}_{\mathbf{t} \mathbf{t}^{\prime}}^{h h^{\prime}} m_{\mathbf{s}+\mathbf{t}}^{h} m_{\mathbf{s}+\mathbf{t}+\mathbf{t}^{\prime}}^{h^{\prime}}+\cdots\right) \\
& \times\langle\mathbf{p}\rangle,
\end{aligned}
$$

where the kernels $\boldsymbol{\alpha}_{\mathbf{t}}^{h}$ and $\boldsymbol{\alpha}_{\mathbf{t t}^{\prime}}^{h h^{\prime}}$ are referred to as the first-order and second-order influence coefficients, respectively, that are assumed to be completely independent of the microstructure descriptors $m_{s}^{h}$. For multiscale problems involving elasticity, these influence coefficients are fourth-rank tensors as shown in (4) (compare also with (2)). The influence coefficients capture the contributions of various microstructure features in the neighborhood of the spatial position $s$ to the local response field at that position. The first-order influence coefficients $\boldsymbol{\alpha}_{\mathbf{t}}^{h}$ capture the influence of the placement of the local state $h$ in a spatial location that is $\mathbf{t}$ away from the spatial cell of interest denoted by s. Likewise, the second-order influence coefficients $\boldsymbol{\alpha}_{\mathbf{t t}^{\prime}}^{h h^{\prime}}$ capture the combined effect of placing local states $h$ and $h^{\prime}$ in spatial cells that are $\mathbf{t}$ and $\mathbf{t}^{\prime}$ away, respectively, from the spatial cell of interest s. In this notation, $\mathbf{t}$ enumerates the bins in the vector space used to define the neighborhood of the spatial bin of interest [90], which has been tessellated using the same scheme that was used for the spatial domain of the material internal structure, that is, $\mathbf{t} \in \mathbf{S}$. It should be noted that the influence coefficients in the localization relationship (4) are closely related to the Green's functions (the precise relationship can be established by comparing (2) and (4)).

Volterra series have been used extensively in the literature to model the dynamic response of nonlinear systems using a set of kernel functions called Volterra kernels [141-146]. The higher-order localization relationship shown in (4) is a particular form of Volterra series and assumes implicitly that the system is spatially invariant, casual, and has finite memory. The influence coefficients in (4) are analogous to Volterra kernels. The higher-order terms in the series are designed to capture systematically the nonlinearity in the system. In other words, the accuracy with which the nonlinearity is captured depends strongly on the specific higherorder terms retained in the localization relationship. The influence coefficients are expected to be independent of the microstructure, since the system is expected to be spatially invariant [13, 125-128].

Several of the initial explorations with the MKS approach $[13,125,127,128]$ utilized only the first-order terms in the series expansion shown in (4). It was generally observed that the first-order terms in the expansion were adequate to capture accurately the microscale distribution of response fields of interest provided the contrast between the possible local states in the microstructure was limited to moderately low values. It was specifically noted that there is a critical need to include the higher-order terms for improving the accuracy of the localization relationships in the MKS framework for material systems with moderate to high contrast. Strong-contrast formulations have been previously utilized 
to predict the effective properties of electrical conductivity $[147,148]$ and elasticity $[17,149]$, but not for predicting the local response fields that are the focus of the MKS framework.

In recent work [126], it was shown that the higher-order terms in (4) can be recast to have the same form as the first-order terms with the use of higher-order microstructure functions that identify specific configurations of local states in a small neighbourhood. More specifically, it was demonstrated that (4) retains the simple form even when the highorder terms are included.

$$
\mathbf{p}_{s}=\left(\sum_{i=1}^{I} \sum_{\mathbf{t} \in \mathbf{S}} \boldsymbol{\alpha}_{t}^{i} m_{\mathbf{s + t}}^{i}\right)\langle\mathbf{p}\rangle .
$$

In (5), the index $i$ now enumerates all of the distinct local configurations, each specified by a selected set of local vectors and the associated local states. The series in (5) is truncated after the consideration of a finite number of such local configurations (denoted by $I$ ).

In prior work $[13,126]$, we have demonstrated that it is possible to estimate the numerical values of the influence coefficients in (4) and (5) by calibration with results obtained from previously established numerical approaches. For example, in problems dealing with elastic deformations in composite materials, the influence coefficients can be established through calibration with results from micromechanical finite-element models. It should be noted that the results from micromechanical finite-element models implicitly satisfy the governing field equations at the mesoscale in the weak numerical sense. Consequently, the MKS metamodels also aim to accomplish the same in their predictions. We have also showed that (4) (and the same applies to (5)) takes a much simpler form when transformed into the discrete Fourier transform (DFT) space, where it can be recast as

$$
\begin{gathered}
\mathbf{P}_{\mathbf{k}}=\left[\left(\sum_{h=1}^{H}\left(\boldsymbol{\beta}_{\mathbf{k}}^{h}\right)^{*} M_{\mathbf{k}}^{h}\right)\right]\langle\mathbf{p}\rangle, \quad \boldsymbol{\beta}_{\mathbf{k}}^{h}=\mathfrak{I}_{\mathbf{k}}\left(\alpha_{\mathbf{t}}^{h}\right), \\
\mathbf{P}_{\mathbf{k}}=\mathfrak{I}_{\mathbf{k}}\left(\mathbf{p}_{\mathbf{s}}\right), \quad M_{\mathbf{k}}^{h}=\mathfrak{I}_{\mathbf{k}}\left(m_{\mathbf{s}}^{h}\right),
\end{gathered}
$$

where $\mathfrak{I}_{\mathbf{k}}()$ denotes the DFT operation with respect to the spatial variables $\mathbf{s}$ or $\mathbf{t}$, and the superscript $*$ denotes the complex conjugate. Note that the number of coupled firstorder coefficients in (6) is only $H$, although the total number of first-order coefficients still remains as $|\mathbf{S}| H$. This simplification is a direct consequence of the well-known convolution properties of DFTs [150]. Because of this dramatic uncoupling of the influence coefficients into smaller sets, it becomes trivial to estimate the values of the influence coefficients $\boldsymbol{\beta}_{\mathrm{k}}^{h}$ by calibrating them against results from numerical models. It is emphasized here that establishing $\boldsymbol{\beta}_{\mathbf{k}}^{h}$ is a one-time computational task for a selected composite material system and a selected physical phenomenon of interest.

The procedures for establishing the influence coefficients have been discussed in detail in our prior work $[13,125-$ 128]. Briefly, the first-order influence coefficients were calibrated using special "delta" microstructures (comprising one voxel of one local state at the center surrounded by voxels of a different local state) on microscale volumes subjected to periodic boundary conditions. For the higher-order influence coefficients, it was necessary to include a larger number of calibration datasets. It is acknowledged here that identifying the correct boundary conditions in multiscale problems is an outstanding problem in the field (see [151] for a discussion of this problem). In our work, we have followed the most commonly employed approach in the literature of using periodic boundary conditions, as they are particularly well suited for DFT representations. It is also noted that the selection of the size of the microscale volume element (MVE) can have a significant influence on the calibrated values of the influence coefficients. Since the influence coefficients are expected to decay to zero values for increasing values of $\mathbf{t}$, the localization captured by (4) is associated with a finite interaction zone or finite memory. In order to capture the spatial characteristics of localization accurately, we recommend that the MVE size used for generating the calibration datasets be at least twice the size of the interaction zone. Since the size of the interaction zone is not known a priori, a few trials are typically needed to establish a suitable MVE size for a given material system and physical phenomenon. In general, we note that the MVE size increases as the contrast in the local properties is increased. Finally, it is also important to ensure that the MVEs are large enough that the boundary conditions do not significantly impact the calibrated values of the influence coefficients.

The influence coefficients established on smaller spatial domains (MVEs) can be easily extended and applied to significantly larger spatial domains $[125,126]$. As noted earlier, we expect the influence functions to decay sharply with increasing $\mathbf{t}$ (just like Green's functions). This allows us to extend the influence functions to larger spatial domains by simply padding the functions with zeros for the larger values of $\mathbf{t}$. The viability of this simple concept has been demonstrated in our recent work [125-127]. It was noted that the trivially extended influence coefficients accurately reproduced the microscale spatial distribution of the desired field on the larger MVEs with about the same accuracy that was realized for the smaller MVEs.

\section{Example Case Study}

In this example, we present the application of the framework described above to the rate-independent rigid-plastic deformation of a two-phase representative volume element (RVE), with no strain hardening [127]. The two phases are assumed to exhibit isotropic plasticity with yield strengths of $200 \mathrm{MPa}$ and $250 \mathrm{MPa}$, respectively. The stress-strain relationships for both phases are assumed to be described by the Levy-Mises equations [152] as

$$
\dot{\varepsilon}=\lambda \sigma^{\prime}
$$

where $\dot{\boldsymbol{\varepsilon}}$ is the symmetric strain rate tensor, $\boldsymbol{\sigma}^{\prime}$ is the symmetric deviatoric Cauchy stress tensor, and $\lambda$ is a proportionality parameter that can be related to the yield strength 
of the material, the equivalent plastic strain rate and the equivalent stress. Although it is not directly apparent from (7), the constitutive relation described implies a rate-independent plastic response. The goal of the localization expression in this example is to compute the local strain rate field in the RVE of the two-phase composite. For simplicity, we shall consider the case of an applied isochoric simple compression strain rate tensor on the RVE at the macroscale, with equal extension in lateral directions, expressed as

$$
\dot{\overline{\boldsymbol{\varepsilon}}}_{i j}=\left[\begin{array}{ccc}
\dot{\bar{\varepsilon}} & 0 & 0 \\
0 & -0.5 \dot{\bar{\varepsilon}} & 0 \\
0 & 0 & -0.5 \dot{\bar{\varepsilon}}
\end{array}\right]
$$

We will subsequently discuss the extension of this localization relationship for other imposed strain rate tensors.

For this example, the local state space is comprised of two isotropic phases $(H=2)$, where $h=1$ identifies the first local state (phase) and $h=2$ identifies the second one. We assume each cell of the tessellated spatial domain to be completely filled with either of the two local states. Thus, the microstructure variable $m_{\mathrm{s}}^{h}$ takes on values of zeros or ones. For instance, if a given cell $s$ is occupied by the first phase, then $m_{\mathrm{s}}^{1}=1$ and $m_{\mathrm{s}}^{2}=0$.

Based on (6), the first-order localization linkage for the present problem can be expressed in the DFT space as

$$
\mathfrak{J}_{\mathbf{k}}\left(\dot{\boldsymbol{\varepsilon}}_{\mathbf{s}}\right)=\left[\sum_{h=1}^{H=2} \boldsymbol{\beta}_{\mathbf{k}}^{h *} M_{\mathbf{k}}^{h}\right] \dot{\bar{\varepsilon}}
$$

where $\dot{\boldsymbol{\varepsilon}}_{\mathrm{s}}$ represents the local strain rate tensor in the spatial bin $\mathbf{s}$, and $\dot{\bar{\varepsilon}}=0.02 \mathrm{~s}^{-1}$ is the macroscopically imposed strain rate in the $\mathbf{e}_{1}$ direction on the RVE (see (8)). It is also important to note that in the notation used in (9), both $\dot{\boldsymbol{\varepsilon}}_{\text {s }}$ and $\boldsymbol{\beta}_{\mathbf{k}}^{* h}$ are second-rank tensors. Introducing the constraints of (3) into (9) results in [13]

$$
\begin{array}{r}
\mathfrak{J}_{\mathbf{k} \neq \mathbf{0}}\left(\dot{\boldsymbol{\varepsilon}}_{\mathbf{s}}\right)=\left[\left(\boldsymbol{\beta}_{\mathbf{k}}^{1 *}-\beta_{\mathbf{k}}^{2 *}\right) M_{\mathbf{k}}^{1}\right] \dot{\bar{\varepsilon}}=\left[\gamma_{\mathbf{k}}^{1} M_{\mathbf{k}}^{1}\right] \dot{\bar{\varepsilon}} \\
\mathfrak{J}_{0}\left(\dot{\boldsymbol{\varepsilon}}_{\mathbf{s}}\right)=|\mathbf{S}|\left(\dot{\bar{\varepsilon}} \mathbf{e}_{1} \otimes \mathbf{e}_{1}-0.5 \dot{\bar{\varepsilon}} \mathbf{e}_{2} \otimes \mathbf{e}_{2}-0.5 \dot{\bar{\varepsilon}} \mathbf{e}_{3} \otimes \mathbf{e}_{3}\right) .
\end{array}
$$

Equation (11) simply indicates that the macroscopic strain rate on the RVE is the same as volume averaged strain rate from the microscale.

It is also important to recognize that if the $\gamma_{\mathrm{k}}^{1}$ are known, the local strain rate field for any given microstructure dataset $m_{\mathrm{s}}^{h}$ subjected to the simple compression loading condition selected here can be computed by applying (10) and (11) performing a simple inverse DFT.

As described earlier, the values of $\gamma_{\mathrm{k}}^{1}$ are established by regression analysis using datasets produced by FE models on selected microstructures. In our work, we discovered that "delta" microstructures, consisting of one element of one phase surrounded completely by another phase, are very convenient for the calibration process and produce the best estimates for $\gamma_{\mathbf{k}}^{1}$. In a two-phase composite, it is possible to define only two distinct delta microstructures, and both of these were used in the calibration process for this problem. This selection is also motivated by the recognition that (10) and
(11) represents a linear and space-invariant causal system. For such systems, when the output for an impulse (i.e., delta microstructure) is known, than the output for any other microstructure input can be described as a convolution of the input with the impulse response [150]. All of the FEM results used in this study were generated using the commercial software ABAQUS, where each RVE contained 804,357 $(93 \times 93 \times 93)$ cuboid-shaped three-dimensional eight-node solid elements. The use of cube-shaped elements naturally defines a regularly spaced grid, conducive to the computation of the DFTs. The macroscale simple compression strain rate was imposed on the finite-element mesh as a periodic uniform boundary condition. The values of $\gamma_{\mathbf{k}}^{1}$ were established as the best-fit values for the FE results on the two delta microstructures described above, using standard linear regression analyses methods [153].

The established $\gamma_{\mathrm{k}}^{1}$ coefficients constitute the materials knowledge systems (MKS) for the case study presented. In fact, the strain rate field for any other RVE comprising any spatial arrangements of the same constituent phases, subjected to the same simple compression loading condition, can be easily computed using (10) and (11). As a critical validation of this concept, we explore the application of the MKS established here to a random microstructure of the selected two phases. We selected a random microstructure for our validation here because their rich diversity of local neighbourhoods produce the most heterogeneous microscale strain rate fields in the composite and, therefore, offer an excellent opportunity to evaluate the localization relationships most critically. We note that we have successfully applied the MKS developed here to a large number of microstructures, although only one example is described here in detail. Figure 1 compares the local $\dot{\varepsilon}_{11}$ component of the strain rate field for the selected random microstructure using both the FE analysis and the MKS approach developed in this work.

The error between the predictions shown in Figure 1 from the MKS approach described here and the FEM analysis can be quantified in each spatial bin as

$$
\operatorname{Err}_{\mathbf{s}}=\left|\frac{\left(\left(\dot{\varepsilon}_{11}\right)_{\mathrm{s}}\right)_{\mathrm{FEM}}-\left(\left(\dot{\varepsilon}_{11}\right)_{\mathrm{s}}\right)_{\mathrm{MKS}}}{\dot{\bar{\varepsilon}}_{11}}\right| \times 100
$$

where the subscripts FEM and MKS indicate that the predictions were made using FEM and MKS methods, respectively. Based on the above definition, the average value of $\operatorname{Err}_{s}$ over all of the spatial bins for the microstructure shown in Figure 1 is only $2.2 \%$. The FE analyses could not be performed on a regular desktop PC. It was executed on an IBM e1350 supercomputing system (part of The Ohio Supercomputer Center) and required 94 processor hours. In contrast, the MKS method took only 32 seconds on a regular laptop (2 GHz CPU and $2 \mathrm{~GB}$ RAM).

In the case study presented here, MKS was developed for a very specific loading condition (i.e., simple compression strain rate tensor). In order to extend the MKS presented here to general loading conditions, we need substantially more 


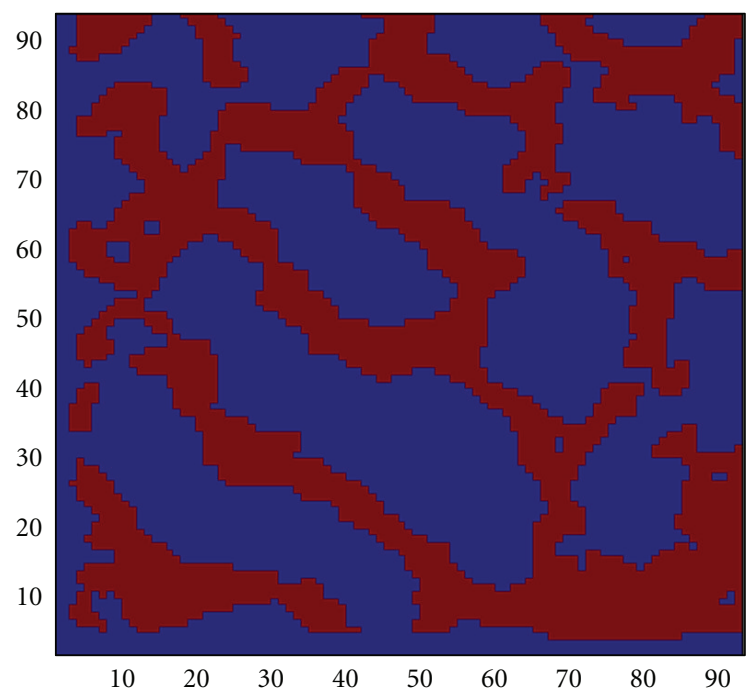

(a)

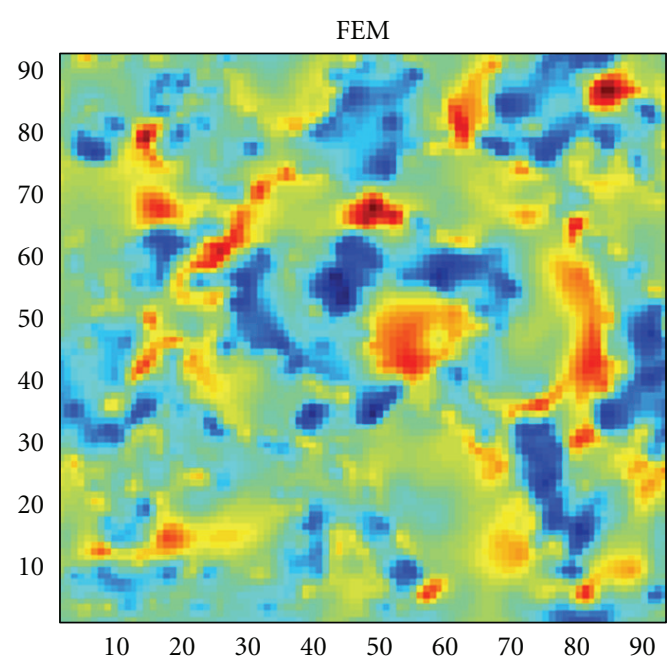

(b)

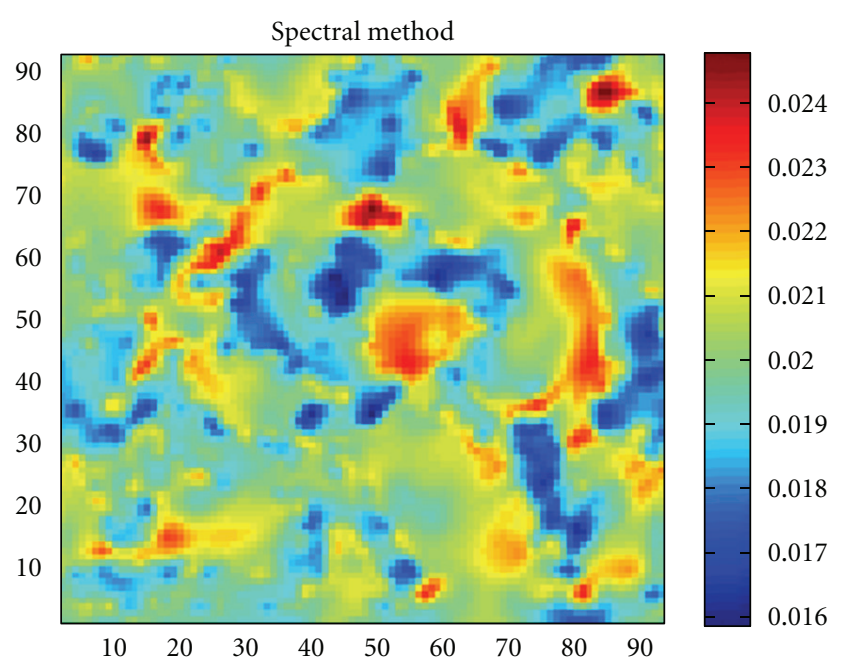

(c)

FIGURE 1: Comparison of the contour maps of the local $\dot{\varepsilon}_{11}$ component of the strain rate tensor for a 3D microstructure. The middle section of the 3D RVE used in the calculation is shown at the top (a), while the predicted strain rate contours by the FE method (b) and the MKS established in this work (c) are shown below. Both phases are assumed to exhibit isotropic plasticity with yield strengths of $200 \mathrm{MPa}$ and $250 \mathrm{MPa}$, respectively. The macroscopic simple compression strain rate applied is $0.02 \mathrm{~s}^{-1}$.

effort. A strategy to address this challenge is outlined below. Towards this end, (9) could be generalized as

$$
\mathfrak{J}_{\mathbf{k}}\left(\dot{\boldsymbol{\varepsilon}}_{\mathbf{s}}\right)=\sum_{h=1}^{H=2} \beta_{\mathbf{k}}^{h *}(\dot{\bar{\varepsilon}}) M_{\mathbf{k}}^{h},
$$

where the dependence of $\beta_{\mathrm{k}}^{h}$ on the macroscale imposed strain rate tensor, $\dot{\bar{\varepsilon}}$, is explicitly noted. We, therefore, need to establish the functional dependence of $\boldsymbol{\beta}_{\mathrm{k}}^{h}$ in the space of symmetric second rank tensors, which is a six-dimensional space. However, if we elect to solve the problem in the principal frame of $\dot{\bar{\varepsilon}}$ (i.e., the microstructure signal needs to be appropriately rotated), then the domain of interest for describing $\beta_{\mathbf{k}}^{h}$ reduces to a three-dimensional space. If we further exploit the fact that the magnitude of $\dot{\bar{\varepsilon}}$ has no effect on the localization (a consequence of the rate independence of the plastic response) and we require $\dot{\bar{\varepsilon}}$ to be traceless (to reflect volume conservation during plastic deformation), then the domain of interest for describing $\beta_{\mathrm{k}}^{h}$ can be expressed using a single angular variable $[154,155]$. The functional dependence of $\beta_{\mathrm{k}}^{h}$ on this single angular variable can be expressed conveniently using DFTs following the approach outlined in our earlier work [155].

As a final comment on this case study, we point out that the composite material studied here had only a low contrast in the properties of the constituent phases (i.e., the yield strengths were $200 \mathrm{MPa}$ and $250 \mathrm{MPa}$, resp.). Based on the various case studies we have successfully completed, it is clear 
that the first-order influence coefficients provide good predictions for composites with low and moderate contrasts. With higher contrasts in the properties of the constituents, it is imperative to include the higher-order terms shown in (4).

\section{Summary and Future Outlook}

MKS is a novel data and computational science enabled mathematical framework for establishing high-fidelity metamodels needed for effective bidirectional scale-bridging in hierarchical multiscale structure-property-processing linkages. The framework is very general and can be applied to a broad range of physical phenomena in a broad range of material systems. This approach exploits the tremendous computational efficiency of FFT- (fast Fourier transforms-) based algorithms for data mining the local structure-response-structure evolution linkages from large numerical datasets produced by established modeling strategies for microscale phenomena. The viability of this new approach has been demonstrated with several case studies involving both linear and nonlinear material responses. It was generally noted that the first-order influence coefficients adequately captured the localization relationships in low-contrast composite systems, in spite of the nonlinearity inherent to the phenomena. It was also observed that the higher-order coefficients are needed with increasing contrast in the local responses of the constituent local states.

An important next step in the development of MKS is its extension to polycrystalline metals. The main challenge in this extension is the fact that the local state in polycrystalline metals will need to include a description of the crystal lattice orientation. In all of the previous applications of the MKS, the composite had a finite number of discrete local states (e.g., the local state space for the composite material in Figure 1 had only two discrete elements, i.e., $H=2$ ). However, when including the lattice orientation in the description of the local state, the corresponding local state space is the continuous orientation space (the space of all distinct proper orthogonal tensors in 3D). Although it is conceivable to discretize this local state space into orientation bins (just like we have discretized the spatial domain into bins in the description of the microstructure function), this approach would be highly inefficient from a computational viewpoint. This is because the number of bins needed to discretize the orientation space is expected to be extremely large. A viable approach to addressing this tremendous challenge lies in recasting the MKS localization relationships ((4) and (5)) in suitable spectral representations for both the spatial variables and the orientation variables. Equation (6) already shows how the spatial variable can be cast in a spectral framework using DFTs. New computationally efficient spectral formulations for the orientation variable need to be explored, possibly through the use of generalized spherical harmonics (GSH) $[9,156]$. For example, the first term in (4) can be rewritten as

$$
\sum_{h=1 \mathbf{t} \in \mathbf{S}}^{H} \boldsymbol{\alpha}_{t}^{h} m_{\mathbf{s}+\mathbf{t}}^{h}=\int_{\mathrm{FZ}} \boldsymbol{\alpha}_{t}(g) f_{\mathbf{s}+\mathbf{t}}(g) d g,
$$

where $g$ denotes the crystal lattice orientation, FZ denotes the fundamental zone of distinct orientations for a selected crystal structure, $f_{s}(g)$ is the orientation distribution function for the spatial bin labeled $\mathbf{s}$, and $d g$ is an invariant measure of orientation space $[9,156]$. Prior work $[9,15,157$, 158] has demonstrated the tremendous advantages of using generalized spherical harmonics (GSH) as a Fourier basis for functions defined on the orientation space. Using the same representations, and exploiting the orthogonal properties of the Fourier basis, (14) can be expressed as

$$
\sum_{h=1 \mathbf{t} \in \mathbf{S}}^{H} \boldsymbol{\alpha}_{t}^{h} m_{\mathbf{s}+\mathbf{t}}^{h}=\sum_{t \ell \mu \nu} \mathbf{A}_{t \ell}^{\mu \nu} \mathbf{F}_{(s+t) \ell}^{\mu \nu *}
$$

where $\mathbf{A}_{t \ell}^{\mu \nu}$ are the Fourier coefficients of $\boldsymbol{\alpha}_{t}(g)$ and $\mathbf{F}_{s \ell}^{\mu \nu}$ are the Fourier coefficients of $f_{\mathbf{s}}(g)$, * denotes a complex conjugate, and indices $\ell, \mu, \nu$ enumerate the generalized spherical harmonics. One of the main advantages of using GSH is that it provides a compact representation for functions of physical tensors over the orientation space. For example, it is already well established that only 10 distinct combinations of $(\ell, \mu, \nu)$ yield nonzero values of $\mathbf{A}_{t \ell}^{\mu \nu}$ for cubic crystal symmetry (can be concluded from results presented in [9, $15,157])$ for elastic localization tensors. This level of dimensionality reduction is of tremendous value in establishing computationally efficient MKS linkages. Note also that the structure of the first term now looks very similar to the first term in (4). We should, therefore, be able to continue to use the DFT approach described earlier for uncoupling the spatial terms (see (6)).

In closing, it is emphasized that the MKS approach allows for a true multiscale simulation of the physical phenomenon with the information flowing in both directions between the constituent length scales [12]. As an example, consider the simulation of a complex processing operation where different macroscale spatial locations in the sample experience different thermal histories (often an unavoidable consequence of the boundary conditions imposed at the macroscale). Consequently, strong variations in the microstructure should be expected at different macroscale locations in the sample. In other words, it is not enough to track the evolution of a single representative microstructure for the entire sample. Furthermore, the development of microstructure heterogeneities can be expected to have a strong influence on the macroscale simulation by altering the local effective properties at different locations in the sample. In such a situation, it is necessary to track evolution of several representative microstructures at various macroscale locations in the sample and effectively pass information in both directions between the microscale simulations and the macroscale simulation. This is extremely difficult, if not impossible, using any of the currently employed techniques. The MKS approach described here offers a viable approach for such problems. For a given problem, it should be possible to set up the necessary MKS linkages as described earlier (this constitutes a one-time computational expense). After the MKS linkages are set up, they can be retrieved with minimal computational expense in a multiscale simulation to not only track accurately the evolution of the microstructure 
at each spatial location of interest in the macroscale simulation, but also to pass their associated updated effective properties influencing the macroscale simulation.

\section{Acknowledgment}

Funding from ONR Award N00014-11-1-0759 (Dr. William M. Mullins, program manager) is gratefully acknowledged.

\section{References}

[1] R. Schafrik, Technology Transition in Aerospace Industry in Workshop on Accelerating Technology Transfer, National Research Council, Washington, DC, USA, 2003.

[2] D. L. McDowell and T. L. Story, "New directions in materials design science and engineering (MDS\&E)," Report of A NSF DMR-Sponsored Workshop, 1998.

[3] Materials Genome Initiative for Global Competitiveness. National Science and Technology Council, Executive Office of the President, 2011.

[4] J. H. Panchal, S. R. Kalidindi, and D. L. McDowell, "Key computational modeling issues in integrated computational materials engineering," Computer-Aided Design, vol. 45, no. 1, pp. 4-25, 2013.

[5] The National Acamedies Press, Integrated Computational Materials Engineering: A TransFormational Discipline For Improved Competitiveness and National Security, The National Acamedies Press, Washington, DC, USA, 2008.

[6] A National Science and Technology Council, A National Strategic Plan For Advanced Manufacturing, National Science and Technology Council, Executive Office of the President, Washington, DC, USA, 2012.

[7] National Science Foundation, Simulation-Based Engineering Science: Revolutionizing Engineering Science Through Simulation, National Science Foundation, Arlington, Va, USA, 2006.

[8] B. L. Adams, S. R. Niezgoda, S. R. Kalidindi, and D. T. Fullwood, "Microstructure sensitive design for performance optimization," Progress in Materials Science, vol. 55, no. 6, pp. 477-562, 2010.

[9] D. T. Fullwood, S. R. Niezgoda, B. L. Adams, and S. R. Kalidindi, "Microstructure sensitive design for performance optimization," Progress in Materials Science, vol. 55, no. 6, pp. 477-562, 2010.

[10] J. R. Willis, "Variational and related methods for the overall properties of composites," Advances in Applied Mechanics C, vol. 21, pp. 1-78, 1981.

[11] O. Sigmund and S. Torquato, "Composites with extremal thermal expansion coefficients," Applied Physics Letters, vol. 69, no. 21, pp. 3203-3205, 1996.

[12] H. F. Al-Harbi, G. Landi, and S. R. Kalidindi, "Multi-scale modeling of the elastic response of a structural component made from a composite material using the materials knowledge system," Modelling and Simulation in Materials Science and Engineering, vol. 20, Article ID 055001, 2012.

[13] G. Landi, S. R. Niezgoda, and S. R. Kalidindi, "Multi-scale modeling of elastic response of three-dimensional voxelbased microstructure datasets using novel DFT-based knowledge systems," Acta Materialia, vol. 58, no. 7, pp. 2716-2725, 2010.

[14] S. Brisard and L. Dormieux, "FFT-based methods for the mechanics of composites: a general variational framework," Computational Materials Science, vol. 49, no. 3, pp. 663-671, 2010.
[15] S. R. Kalidindi, G. Landi, and D. T. Fullwood, "Spectral representation of higher-order localization relationships for elastic behavior of polycrystalline cubic materials," Acta Materialia, vol. 56, no. 15, pp. 3843-3853, 2008.

[16] M. Binci, D. Fullwood, and S. R. Kalidindi, "A new spectral framework for establishing localization relationships for elastic behavior of composites and their calibration to finiteelement models," Acta Materialia, vol. 56, no. 10, pp. 22722282, 2008.

[17] D. T. Fullwood, B. L. Adams, and S. R. Kalidindi, "A strong contrast homogenization formulation for multi-phase anisotropic materials," Journal of the Mechanics and Physics of Solids, vol. 56, no. 6, pp. 2287-2297, 2008.

[18] G. W. Milton, "The theory of composites," in Cambridge Monographs on Applied and Computational Mathematics, P. G. Ciarlet, Ed., Cambridge university press, Cambridge, Uk, 2002.

[19] B. L. Adams and T. Olson, "The mesostructure-properties linkage in polycrystals," Progress in Materials Science, vol. 43, no. 1, pp. 1-88, 1998.

[20] B. P. Flannery, H. W. Deckman, W. G. Roberge, and K. L. D'Amico, "Three-dimensional x-ray microtomography," Science, vol. 237, no. 4821, pp. 1439-1444, 1987.

[21] E. Maire, J. Y. Buffire, L. Salvo, J. J. Blandin, W. Ludwig, and J. M. Ltang, "On the application of X-ray microtomography in the field of materials science," Advanced Engineering Materials, vol. 3, no. 8, pp. 539-546, 2001.

[22] J. Alkemper and P. W. Voorhees, "Quantitative serial sectioning analysis," Journal of Microscopy, vol. 201, no. 3, pp. 388394, 2001.

[23] V. G. M. Sivel, J. Van Den Brand, W. R. Wang et al., "Application of the dual-beam FIB/SEM to metals research," Journal of Microscopy, vol. 214, no. 3, pp. 237-245, 2004.

[24] D. Blavette, A. Bostel, J. M. Sarrau, B. Deconihout, and A. Menand, "An atom probe for three-dimensional tomography," Nature, vol. 363, no. 6428, pp. 432-438, 1993.

[25] D. N. Seidman, "Three-dimensional atom-probe tomography: advances and applications," Annual Review of Materials Research, vol. 37, pp. 127-158, 2007.

[26] M. D. Uchic, M. De Graef, R. Wheeler, and D. M. Dimiduk, "Microstructural tomography of a $\mathrm{Ni}_{70} \mathrm{Cr}_{20} \mathrm{Al}_{10}$ superalloy using focused ion beam microscopy," Ultramicroscopy, vol. 109, no. 10, pp. 1229-1235, 2009.

[27] R. Srinivasan, R. Banerjee, J. Y. Hwang et al., "Atomic scale structure and chemical composition across order-disorder interfaces," Physical Review Letters, vol. 102, no. 8, Article ID 086101, 2009.

[28] A. Stiénon, A. Fazekas, J. Y. Buffière, A. Vincent, P. Daguier, and F. Merchi, "A new methodology based on X-ray microtomography to estimate stress concentrations around inclusions in high strength steels," Materials Science and Engineering A, vol. 513-514, pp. 376-383, 2009.

[29] M. K. Miller and R. G. Forbes, "Atom probe tomography," Materials Characterization, vol. 60, no. 6, pp. 461-469, 2009.

[30] O. Betz, U. G. K. Wegst, D. Weide et al., "Imaging applications of Synchrotron $\mathrm{x}$-ray micro-tomography in biological morphology and biomaterial science. I. General aspects of the technique and its advantages in the analysis of arthropod structures," Journal of Microscopy, vol. 227, no. 1, pp. 51-71, 2007.

[31] M. D. Uchic, L. Holzer, B. J. Inkson, E. L. Principe, and P. Munroe, "Three-dimensional microstructural characterization using focused ion beam tomography," MRS Bulletin, vol. 32, no. 5, pp. 408-416, 2007. 
[32] H. Proudhon, J. Y. Buffière, and S. Fouvry, "Three-dimensional study of a fretting crack using synchrotron X-ray micro-tomography," Engineering Fracture Mechanics, vol. 74, no. 5, pp. 782-793, 2007.

[33] I. Tiseanu, T. Craciunescu, T. Petrisor, and A. D. Corte, “3D X-ray micro-tomography for modeling of NB3SN multifilamentary superconducting wires," Fusion Engineering and Design, vol. 82, no. 5-14, pp. 1447-1453, 2007.

[34] L. Holzer, F. Indutnyi, P. Gasser, B. Münch, and M. Wegmann, "Three-dimensional analysis of porous $\mathrm{BaTiO}_{3}$ ceramics using FIB nanotomography," Journal of Microscopy, vol. 216, no. 1, pp. 84-95, 2004.

[35] L. Salvo, P. Cloetens, E. Maire et al., "X-ray micro-tomography an attractive characterisation technique in materials science," Nuclear Instruments and Methods in Physics Research B, vol. 200, pp. 273-286, 2003.

[36] P. A. Midgley and M. Weyland, "3D electron microscopy in the physical sciences: the development of Z-contrast and EFTEM tomography," Ultramicroscopy, vol. 96, no. 3-4, pp. 413-431, 2003.

[37] R. A. Brockman, A. L. Pilchak, W. John Porter, and R. John, "Estimation of grain boundary diffusivity in near- $\alpha$ titanium polycrystals," Scripta Materialia, vol. 65, no. 6, pp. 513-515, 2011.

[38] T. A. Parthasarathy, W. J. Porter, S. Boone, R. John, and P. Martin, "Life prediction under tension of titanium alloys that develop an oxygenated brittle case during use," Scripta Materialia, vol. 65, no. 5, pp. 420-423, 2011.

[39] D. J. Rowenhorst, A. C. Lewis, and G. Spanos, "Threedimensional analysis of grain topology and interface curvature in a $\beta$-titanium alloy," Acta Materialia, vol. 58 , no. 16, pp. 5511-5519, 2010.

[40] B. S. Fromm, B. L. Adams, S. Ahmadi, and M. Knezevic, "Grain size and orientation distributions: application to yielding of $\alpha$-titanium," Acta Materialia, vol. 57, no. 8, pp. 2339-2348, 2009.

[41] Y. W. Kim, A. Rosenberger, and D. M. Dimiduk, "Microstructural changes and estimated strengthening contributions in a gamma alloy Ti-45Al-5Nb pack-rolled sheet," Intermetallics, vol. 17, no. 12, pp. 1017-1027, 2009.

[42] S. K. Jha, R. John, and J. M. Larsen, "Nominal vs local shot-peening effects on fatigue lifetime in Ti-6Al-2Sn-4Zr$6 \mathrm{Mo}$ at elevated temperature," Metallurgical and Materials Transactions A, vol. 40, no. 11, pp. 2675-2684, 2009.

[43] J. J. Ruschau, R. John, S. R. Thompson, and T. Nicholas, "Fatigue crack growth rate characteristics of Laser Shock Peened Ti-6Al-4V," Journal of Engineering Materials and Technology, Transactions of the ASME, vol. 121, no. 3, pp. 321329, 1999.

[44] S. R. Kalidindi, S. R. Niezgoda, and A. A. Salem, "Microstructure informatics using higher-order statistics and efficient data-mining protocols," Journal of Management, vol. 63, no. 4, pp. 34-41, 2011.

[45] A. A. Salem, S. R. Kalidindi, R. D. Doherty, and S. L. Semiatin, "Strain hardening due to deformation twinning in $\alpha$-titanium: mechanisms," Metallurgical and Materials Transactions A, vol. 37, no. 1, pp. 259-268, 2006.

[46] X. Wu, S. R. Kalidindi, C. Necker, and A. A. Salem, "Modeling anisotropic stress-strain response and crystallographic texture evolution in $\alpha$-titanium during large plastic deformation using taylor-type models: influence of initial texture and purity," Metallurgical and Materials Transactions A, vol. 39, no. 12, pp. 3046-3054, 2008.
[47] X. Wu, S. R. Kalidindi, C. Necker, and A. A. Salem, "Prediction of crystallographic texture evolution and anisotropic stress-strain curves during large plastic strains in high purity $\alpha$-titanium using a Taylor-type crystal plasticity model," Acta Materialia, vol. 55, no. 2, pp. 423-432, 2007.

[48] D. M. Dimiduk, "Gamma titanium aluminide alloys-an assessment within the competition of aerospace structural materials," Materials Science and Engineering A, vol. 263, no. 2, pp. 281-288, 1999.

[49] D. M. Dimiduk, "Systems engineering of gamma titanium aluminides: impact of fundamentals on development strategy," Intermetallics, vol. 6, no. 7-8, pp. 613-621, 1998.

[50] D. M. Dimiduk, P. L. Martin, and Y. W. Kim, "Microstructure development in gamma alloy mill products by thermomechanical processing," Materials Science and Engineering A, vol. 243, no. 1-2, pp. 66-76, 1998.

[51] D. M. Dimiduk, P. M. Hazzledine, T. A. Parthasarathy, S. Seshagiri, and M. G. Mendiratta, "The role of grain size and selected microstructural parameters in strengthening fully lamellar TiAl alloys," Metallurgical and Materials Transactions A, vol. 29, no. 1, pp. 37-47, 1998.

[52] S. L. Semiatin, V. Seetharaman, D. M. Dimiduk, and K. H. G. Ashbee, "Phase transformation behavior of gamma titanium aluminide alloys during supertransus heat treatment," Metallurgical and Materials Transactions A, vol. 29, no. 1, pp. 7-18, 1998.

[53] D. M. Dimiduk, D. B. Miracle, and C. H. Ward, "Development of intermetallic materials for aerospace systems," Materials Science and Technology, vol. 8, no. 4, pp. 367-375, 1992.

[54] Y. W. Kim and D. M. Dimiduk, "Progress in the understanding of gamma titanium aluminides," Journal of Management, vol. 43, no. 8, pp. 40-47, 1991.

[55] M. Khaleel, "Characterization of thermo-mechanical behaviors of advanced high strength steels (AHSS): formability, weldability and performance evaluations of AHSS parts for automotive structures, in Pacific Northwest National Laboratory FY, 2006," Annual Report, Pacific Northwest National Laboratory, Richland, Wash, USA, 2006.

[56] G. Frommeyer, U. Brüx, and P. Neumann, "Supra-ductile and high-strength manganese-TRIP/TWIP steels for high energy absorption purposes," ISIJ International, vol. 43, no. 3, pp. 438-446, 2003.

[57] M. Cherkaoui, M. Berveiller, and X. Lemoine, "Couplings between plasticity and martensitic phase transformation: overall behavior of polycrystalline TRIP steels," International journal of plasticity, vol. 16, no. 10, pp. 1215-1241, 2000.

[58] O. Grassel, L. Kruger, G. Frommeyer, and L. W. Meyer, "High strength $\mathrm{Fe}-\mathrm{Mn}-(\mathrm{Al}, \mathrm{Si})$ TRIP/TWIPsteels developmentproperties-application," International Journal of Plasticity, vol. 16, no. 10, pp. 1391-1409, 2000.

[59] S. Widge, Comparison of Carpenter CusTom 465 Stainless To High Performance Structural Alloys and Other PH Stainless Steels, ShipTech, Biloxi, Miss, USA, 2007.

[60] A. S. Hamada, L. P. Karjalainen, M. C. Somani, and R. M. Ramadan, "Deformation mechanisms in high-al bearing high-Mn TWIP Steels in hot compression and in tension at low temperatures," Materials Science Forum, vol. 550, pp. 217-222, 2007.

[61] B. Gardey, S. Bouvier, V. Richard, and B. Bacroix, "Texture and dislocation structures observation in a dual-phase steel under strain-path changes at large deformation," Materials Science and Engineering A, vol. 400-401, no. 1-2, pp. 136-141, 2005. 
[62] C. L. Xie, S. Ghosh, and M. Groeber, "Modeling cyclic deformation of HSLA steels using crystal plasticity," Journal of Engineering Materials and Technology, Transactions of the ASME, vol. 126, no. 4, pp. 339-352, 2004.

[63] S. Hao, B. Moran, W. K. Liu, and G. B. Olson, "A hierarchical multi-physics model for design of high toughness steels," Journal of Computer-Aided Materials Design, vol. 10, no. 2, pp. 99-142, 2003.

[64] C. E. Campbell and G. B. Olson, "Systems design of high performance stainless steels I. Conceptual and computational design," Journal of Computer-Aided Materials Design, vol. 7, no. 3, pp. 145-170, 2000.

[65] C. E. Campbell and G. B. Olson, "Systems design of high performance stainless steels II. Prototype characterization," Journal of Computer-Aided Materials Design, vol. 7, no. 3, pp. 171-194, 2000.

[66] G. B. Olson, "Systems design of hierarchically structured materials: advanced steels," Journal of Computer-Aided Materials Design, vol. 4, no. 3, pp. 143-156, 1998.

[67] E. Martin, L. Capolungo, L. Jiang, and J. J. Jonas, "Variant selection during secondary twinning in Mg-3\%Al," Acta Materialia, 2010.

[68] G. Proust, C. N. Tomé, A. Jain, and S. R. Agnew, "Modeling the effect of twinning and detwinning during strain-path changes of magnesium alloy AZ31," International Journal of Plasticity, vol. 25, no. 5, pp. 861-880, 2009.

[69] D. L. Yin, J. T. Wang, J. Q. Liu, and X. Zhao, "On tensioncompression yield asymmetry in an extruded Mg-3Al-1Zn alloy," Journal of Alloys and Compounds, vol. 478, no. 1-2, pp. 789-795, 2009.

[70] Y. Chino, K. Kimura, and M. Mabuchi, "Twinning behavior and deformation mechanisms of extruded AZ31 Mg alloy," Materials Science and Engineering A, vol. 486, no. 1-2, pp. 481-488, 2008.

[71] S. H. Choi, J. K. Kim, B. J. Kim, and Y. B. Park, "The effect of grain size distribution on the shape of flow stress curves of Mg-3Al-1Zn under uniaxial compression," Materials Science and Engineering A, vol. 488, no. 1-2, pp. 458-467, 2008.

[72] M. Hakamada, T. Furuta, Y. Chino, Y. Chen, H. Kusuda, and M. Mabuchi, "Life cycle inventory study on magnesium alloy substitution in vehicles," Energy, vol. 32, no. 8, pp. 13521360, 2007.

[73] L. Jiang, J. J. Jonas, R. K. Mishra, A. A. Luo, A. K. Sachdev, and S. Godet, "Twinning and texture development in two Mg alloys subjected to loading along three different strain paths," Acta Materialia, vol. 55, no. 11, pp. 3899-3910, 2007.

[74] M. R. Barnett, M. D. Nave, and C. J. Bettles, "Deformation microstructures and textures of some cold rolled Mg alloys," Materials Science and Engineering A, vol. 386, no. 1-2, pp. 205-211, 2004.

[75] M. Knezevic, A. Levinson, R. Harris, R. K. Mishra, R. D. Doherty, and S. R. Kalidindi, "Deformation twinning in AZ31: influence on strain hardening and texture evolution," Acta Materialia, vol. 58, no. 19, pp. 6230-6242, 2010.

[76] A. Levinson, R. K. Mishra, R. D. Doherty, and S. R. Kalidindi, "Microstructure evolution during roller hemming of AZ31B magnesium sheet," Metallurgical and Materials Transactions A, vol. 43, no. 10, pp. 3824-3833, 2012.

[77] J. E. Carsley, Warm Bending Magnesium Sheet For Automotive Closure Panels, MS\&T, Pittsburgh, Pa, USA, 2009.

[78] Y.-N. Lin, H. Y. Wu, G. Z. Zhou, C. H. Chiu, and S. Lee, "Mechanical and anisotropic behaviors of Mg-Li-Zn alloy thin sheets," Materials and Design, vol. 29, no. 10, pp. 20612065, 2008.

[79] T. Al-Samman and G. Gottstein, "Room temperature formability of a magnesium AZ31 alloy: examining the role of texture on the deformation mechanisms," Materials Science and Engineering A, vol. 488, no. 1-2, pp. 406-414, 2008.

[80] S. V. S. N. Murty, M. C. Mittal, and P. P. Sinha, "Development of high strength bulk ultrafine-grained magnesium alloy AZ31 by multi-pass warm rolling," in Proceedings of the International Conference on Aerospace Science and Technology, Bangalore, India, 2008.

[81] J. Carsley and S. Kim, "Warm hemming of magnesium sheet," Journal of Materials Engineering and Performance, vol. 16, no. 3, pp. 331-338, 2007.

[82] Magnesium Vision 2020: A North American Automotive Strategic Vision for Magnesium, in United States Automotive Materials Partnership, 2006.

[83] B. L. Mordike and T. Ebert, "Magnesium properties-applications-potential," Materials Science and Engineering A, vol. 302, no. 1, pp. 37-45, 2001.

[84] B. Plunkett, R. A. Lebensohn, O. Cazacu, and F. Barlat, "Anisotropic yield function of hexagonal materials taking into account texture development and anisotropic hardening," Acta Materialia, vol. 54, no. 16, pp. 4159-4169, 2006.

[85] C. N. Tomé, R. A. Lebensohn, and U. F. Kocks, "A model for texture development dominated by deformation twinning: application to zirconium alloys," Acta Metallurgica Et Materialia, vol. 39, no. 11, pp. 2667-2680, 1991.

[86] B. L. Adams and K. L. Murty, "Biaxial creep of textured zircaloy II: crystal mean plastic modelling," Materials Science and Engineering C, vol. 70, pp. 181-190, 1985.

[87] B. L. Adams and K. L. Murty, "Creep modeling of textured ziraloy under biaxial sressing. in Mechanical Behaviour of Materials-4," in Proceedings of the 4th International Conference on Flexible Manufacturing Systems, Pergamon Press, Stockholm, Sweden, 1984.

[88] G. S. Clevinger, B. L. Adams, and K. L. Murty, "Analysis of irradiation growth and multiaxial deformation behavior of nuclear fuel cladding," in Proceedings of the 4th International Conference on Structural Mechanics in Reactor Technology, Jun 26-29 1978, pp. 189-201, ASTM, Philadelphia, Pa, USA, 1979.

[89] S. Torquato, Random Heterogeneous Materials, SpringerVerlag, New York, NY, USA, 2002.

[90] B. L. Adams, X. Gao, and S. R. Kalidindi, "Finite approximations to the second-order properties closure in single phase polycrystals," Acta Materialia, vol. 53, no. 13, pp. 3563-3577, 2005.

[91] T. A. Mason and B. L. Adams, "Use of microstructural statistics in predicting polycrystalline material properties," Metallurgical and Materials Transactions A, vol. 30, no. 4, pp. 969-979, 1999.

[92] G. Saheli, H. Garmestani, and B. L. Adams, "Microstructure design of a two phase composite using two-point correlation functions," Journal of Computer-Aided Materials Design, vol. 11, no. 2-3, pp. 103-115, 2005.

[93] M. J. Beran, T. A. Mason, B. L. Adams, and T. Olsen, "Bounding elastic constants of an orthotropic polycrystal using measurements of the microstructure," Journal of the Mechanics and Physics of Solids, vol. 44, no. 9, pp. 1543-1563, 1996.

[94] J. Zeman and M. Šejnoha, "From random microstructures to representative volume elements," Modelling and Simulation in Materials Science and Engineering, vol. 15, no. 4, pp. S325S335, 2007. 
[95] S. Sankaran and N. Zabaras, "A maximum entropy approach for property prediction of random microstructures," Acta Materialia, vol. 54, no. 8, pp. 2265-2276, 2006.

[96] A. Tewari, A. M. Gokhale, J. E. Spowart, and D. B. Miracle, "Quantitative characterization of spatial clustering in threedimensional microstructures using two-point correlation functions," Acta Materialia, vol. 52, no. 2, pp. 307-319, 2004.

[97] A. A. Gusev, P. J. Hine, and I. M. Ward, "Fiber packing and elastic properties of a transversely random unidirectional glass/epoxy composite," Composites Science and Technology, vol. 60, no. 4, pp. 535-541, 2000.

[98] A. A. Gusev, "Representative volume element size for elastic composites: a numerical study," Journal of the Mechanics and Physics of Solids, vol. 45, no. 9, pp. 1449-1459, 1997.

[99] S. Nemat-Nasser and M. Hori, Micromechanics: Overall Properties of Heterogeneous Materials, Elsevier, Amsterdam, The Netherlands, 2nd edition, 1999.

[100] J. McCoy, "Macroscopic response of continua with random microstructures," in Mechanics Today, vol. 6, 1981.

[101] U. Hornung, Homogenization and Porous Media, vol. 6 of Interdisciplinary Applied Mathematics, Springer, New York, NY, USA, 1997.

[102] A. Cherkaev, Variational Methods For Structural Optimization, vol. 140 of Interdisciplinary Applied Mathematics, Springer, New York, NY, USA, 1991.

[103] X. L. Chen and Y. J. Liu, "Square representative volume elements for evaluating the effective material properties of carbon nanotube-based composites," Computational Materials Science, vol. 29, no. 1, pp. 1-11, 2004.

[104] W. J. Drugan and J. R. Willis, "A micromechanics-based nonlocal constitutive equation and estimates of representative volume element size for elastic composites," Journal of the Mechanics and Physics of Solids, vol. 44, no. 4, pp. 497-524, 1996.

[105] T. Kanit, S. Forest, I. Galliet, V. Mounoury, and D. Jeulin, "Determination of the size of the representative volume element for random composites: statistical and numerical approach," International Journal of Solids and Structures, vol. 40, no. 13-14, pp. 3647-3679, 2003.

[106] Z. Shan and A. M. Gokhale, "Representative volume element for non-uniform micro-structure," Computational Materials Science, vol. 24, no. 3, pp. 361-379, 2002.

[107] W. F. Brown, "Solid mixture permittivities," The Journal of Chemical Physics, vol. 23, no. 8, pp. 1514-1517, 1955.

[108] M. J. Beran, Statistical Continuum Theories, John Wiley \& Sons, Interscience, New York, NY, USA, 1968.

[109] S. R. Niezgoda, Y. C. Yabansu, and S. R. Kalidindi, "Understanding and visualizing microstructure and microstructure variance as a stochastic process," Acta Materialia, 2011.

[110] S. R. Niezgoda, D. M. Turner, D. T. Fullwood, and S. R. Kalidindi, "Optimized structure based representative volume element sets reflecting the ensemble-averaged 2-point statistics," Acta Materialia, vol. 58, no. 13, pp. 4432-4445, 2010.

[111] D. L. McDowell, S. Ghosh, and S. R. Kalidindi, "Representation and computational structure-property relations of random media," Journal of Management, vol. 63, no. 3, pp. 45-51, 2011.

[112] S. M. Qidwai, D. M. Turner, S. R. Niezgoda et al., "Estimating response of polycrystalline materials using sets of weighted statistical volume elements (WSVEs)," Acta Materialia, vol. 60, no. 13-14, pp. 5284-5299, 2012.

[113] S. Ghosh, Z. Nowak, and L. Kyunghoon, "Quantitative characterization and modeling of composite microstructures by Voronoi cells," Acta Materialia, vol. 45, no. 6, pp. 22152234, 1997.

[114] S. Ghosh, K. Lee, and S. Moorthy, "Multiple scale analysis of heterogeneous elastic structures using homogenization theory and voronoi cell finite element method," International Journal of Solids and Structures, vol. 32, no. 1, pp. 27-62, 1995.

[115] V. G. Kouznetsova, M. G. D. Geers, and W. A. M. Brekelmans, "Multi-scale second-order computational homogenization of multi-phase materials: a nested finite element solution strategy," Computer Methods in Applied Mechanics and Engineering, vol. 193, no. 48-51, pp. 5525-5550, 2004.

[116] V. Kouznetsova, M. G. D. Geers, and W. A. M. Brekelmans, "Multi-scale constitutive modelling of heterogeneous materials with a gradient-enhanced computational homogenization scheme," International Journal for Numerical Methods in Engineering, vol. 54, no. 8, pp. 1235-1260, 2002.

[117] H. Kadowaki and W. K. Liu, "Bridging multi-scale method for localization problems," Computer Methods in Applied Mechanics and Engineering, vol. 193, no. 30-32, pp. 32673302, 2004.

[118] D. L. McDowell, H. J. Choi, J. Panchal, R. Austin, J. Allen, and F. Mistree, "Plasticity-related microstructure-property relations for materials design," Key Engineering Materials, vol. 340-341, pp. 21-30, 2007.

[119] H. J. Choi, D. L. McDowell, J. K. Allen, and F. Mistree, "An inductive design exploration method for hierarchical systems design under uncertainty," Engineering Optimization, vol. 40, no. 4, pp. 287-307, 2008.

[120] D. J. Luscher, D. L. McDowell, and C. A. Bronkhorst, "A second gradient theoretical framework for hierarchical multiscale modeling of materials," International Journal of Plasticity, vol. 26, no. 8, pp. 1248-1275, 2010.

[121] G. B. Olson, "Computational design of hierarchically structured materials," Science, vol. 277, no. 5330, pp. 1237-1242, 1997.

[122] T. Nicholas, A. Hutson, R. John, and S. Olson, "A fracture mechanics methodology assessment for fretting fatigue," International Journal of Fatigue, vol. 25, no. 9-11, pp. 10691077, 2003.

[123] H. Choi, D. L. McDowell, J. K. Allen, D. Rosen, and F. Mistree, "An inductive design exploration method for robust multiscale materials design," Journal of Mechanical Design, Transactions of the ASME, vol. 130, no. 3, Article ID 031402, 2008.

[124] D. L. McDowell, J. H. Panchal, H. J. Choi, C. C. Seepersad, J. K. Allen, and F. Mistree, Integrated Design of Multiscale, Multifunctional Materials and Products, Elsevier, New York, NY, USA, 2009.

[125] T. Fast, S. R. Niezgoda, and S. R. Kalidindi, "A new framework for computationally efficient structure-structure evolution linkages to facilitate high-fidelity scale bridging in multi-scale materials models," Acta Materialia, vol. 59, no. 2, pp. 699707, 2011.

[126] T. Fast and S. R. Kalidindi, "Formulation and calibration of higher-order elastic localization relationships using the MKS approach," Acta Materialia, vol. 59, no. 11, pp. 4595-4605, 2011.

[127] S. R. Kalidindi, S. R. Niezgoda, G. Landi, S. Vachhani, and T. Fast, "A novel framework for building materials knowledge systems," Computers, Materials and Continua, vol. 17, no. 2, pp. 103-125, 2010.

[128] G. Landi and S. R. Kalidindi, "Thermo-elastic localization relationships for multi-phase composites," Computers, Materials and Continua, vol. 16, no. 3, pp. 273-293, 2010. 
[129] E. Kroner, "Statistical modelling," in Modelling Small Deformations of Polycrystals, J. Gittus and J. Zarka, Eds., pp. 229291, Elsevier Science Publishers, London, UK, 1986.

[130] E. Kröner, "Bounds for effective elastic moduli of disordered materials," Journal of the Mechanics and Physics of Solids, vol. 25, no. 2, pp. 137-155, 1977.

[131] S. R. Kalidindi, M. Binci, D. Fullwood, and B. L. Adams, "Elastic properties closures using second-order homogenization theories: case studies in composites of two isotropic constituents," Acta Materialia, vol. 54, no. 11, pp. 3117-3126, 2006.

[132] J. C. Michel, H. Moulinec, and P. Suquet, "Effective properties of composite materials with periodic microstructure: a computational approach," Computer Methods in Applied Mechanics and Engineering, vol. 172, no. 1-4, pp. 109-143, 1999.

[133] H. Moulinec and P. Suquet, "A numerical method for computing the overall response of nonlinear composites with complex microstructure," Computer Methods in Applied Mechanics and Engineering, vol. 157, no. 1-2, pp. 69-94, 1998.

[134] J. Zeman, J. Vondřejc, J. Novák, and I. Marek, "Accelerating a FFT-based solver for numerical homogenization of periodic media by conjugate gradients," Journal of Computational Physics, vol. 229, no. 21, pp. 8065-8071, 2010.

[135] R. A. Lebensohn, "N-site modeling of a 3D viscoplastic polycrystal using Fast Fourier Transform," Acta Materialia, vol. 49, no. 14, pp. 2723-2737, 2001.

[136] R. A. Lebensohn, A. K. Kanjarla, and P. Eisenlohr, "An elastoviscoplastic formulation based on fast Fourier transforms for the prediction of micromechanical fields in polycrystalline materials," International Journal of Plasticity, vol. 32-33, pp. 59-69, 2012.

[137] S. B. Lee, R. A. Lebensohn, and A. D. Rollett, "Modeling the viscoplastic micromechanical response of two-phase materials using Fast Fourier Transforms," International Journal of Plasticity, vol. 27, no. 5, pp. 707-727, 2011.

[138] S. R. Niezgoda and S. R. Kalidindi, "Applications of the phase-coded generalized hough transform to feature detection, analysis, and segmentation of digital microstructures," Computers Materials and Continua, vol. 14, no. 2, pp. 79-97, 2009.

[139] D. T. Fullwood, S. R. Niezgoda, and S. R. Kalidindi, "Microstructure reconstructions from 2-point statistics using phaserecovery algorithms," Acta Materialia, vol. 56, no. 5, pp. 942948, 2008.

[140] D. T. Fullwood, S. R. Kalidindi, S. R. Niezgoda, A. Fast, and N. Hampson, "Gradient-based microstructure reconstructions from distributions using fast Fourier transforms," Materials Science and Engineering A, vol. 494, no. 1-2, pp. 68-72, 2008.

[141] J. A. Cherry, Distortion Analysis of Weakly Nonlinear Filters Using Volterra Series, National Library of Canada, Ottawa, Canada, 1995.

[142] V. Volterra, Theory of Functionals and Integral and IntegroDifferential Equations, Dover, New York, NY, USA, 1959.

[143] N. Wiener, Nonlinear Problems in Random Theory, MIT Press, Cambridge, Mass, USA, 1958.

[144] J. Wray and G. G. R. Green, "Calculation of the Volterra kernels of non-linear dynamic systems using an artificial neural network," Biological Cybernetics, vol. 71, no. 3, pp. 187-195, 1994.

[145] M. J. Korenberg and I. W. Hunter, "The identification of nonlinear biological systems: volterra kernel approaches," Annals of Biomedical Engineering, vol. 24, no. 2, pp. 250-268, 1996.
[146] Y. W. Lee and M. Schetzen, "Measurement of the Wiener Kernels of a non-linear system by cross-correlation," International Journal of Control, vol. 2, no. 3, pp. 237-254, 1965.

[147] S. Torquato, "Effective electrical conductivity of two-phase disordered composite media," Journal of Applied Physics, vol. 58, no. 10, pp. 3790-3797, 1985.

[148] W. F. Brown, "Solid mixture permittivities," The Journal of Chemical Physics, vol. 23, no. 8, pp. 1514-1517, 1955.

[149] S. Torquato, "Effective stiffness tensor of composite media-I. Exact series expansions," Journal of the Mechanics and Physics of Solids, vol. 45, no. 9, pp. 1421-1444, 1997.

[150] A. V. Oppenheim, R. W. Schafer, and J. R. Buck, Discrete Time Signal Processing, Prentice Hall, Englewood Cliffs, NJ, USA, 1999.

[151] S. D. Mesarovic and J. Padbidri, "Minimal kinematic boundary conditions for simulations of disordered microstructures," Philosophical Magazine, vol. 85, no. 1, pp. 65-78, 2005.

[152] A. S. Khan and S. Huang, Continuum Theory of Plasticity, Wiley, New York, NY, USA, 1995.

[153] D. C. Montgomery, E. A. Peck, and G. G. Vining, Introduction To Linear Regression Analysis, Wiley-Interscience, Hoboken, NJ, USA, 2006.

[154] P. Van Houtte, "Application of plastic potentials to strain rate sensitive and insensitive anisotropic materials," International Journal of Plasticity, vol. 10, no. 7, pp. 719-748, 1994.

[155] M. Knezevic, H. F. Al-Harbi, and S. R. Kalidindi, "Crystal plasticity simulations using discrete Fourier transforms," Acta Materialia, vol. 57, no. 6, pp. 1777-1784, 2009.

[156] H.-J. Bunge, Texture Analysis in Materials Science. Mathematical Methods, Cuvillier Verlag, Göttingen, Germany, 1993.

[157] G. Proust and S. R. Kalidindi, "Procedures for construction of anisotropic elastic-plastic property closures for face-centered cubic polycrystals using first-order bounding relations," Journal of the Mechanics and Physics of Solids, vol. 54, no. 8, pp. 1744-1762, 2006.

[158] S. R. Kalidindi, H. K. Duvvuru, and M. Knezevic, "Spectral calibration of crystal plasticity models," Acta Materialia, vol. 54, no. 7, pp. 1795-1804, 2006. 

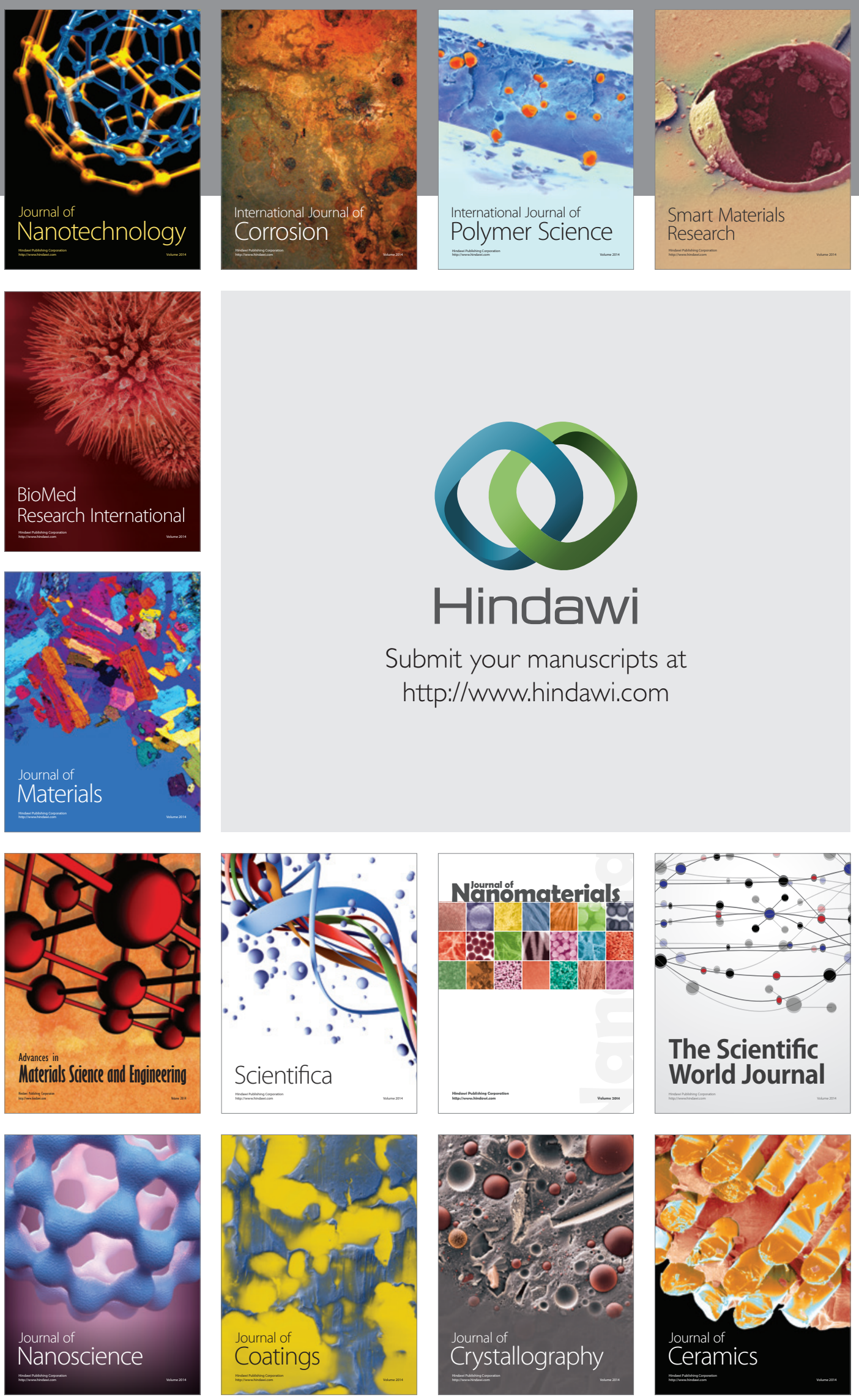

The Scientific World Journal

Submit your manuscripts at

http://www.hindawi.com

\section{World Journal}

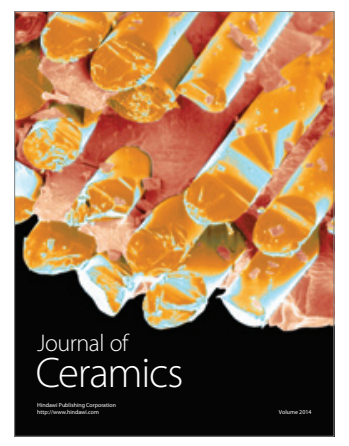

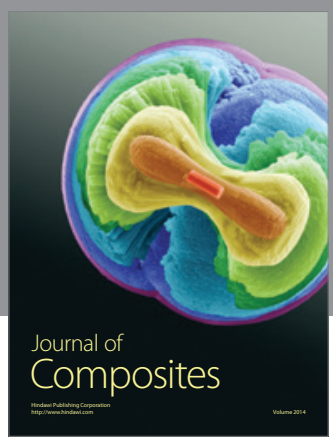
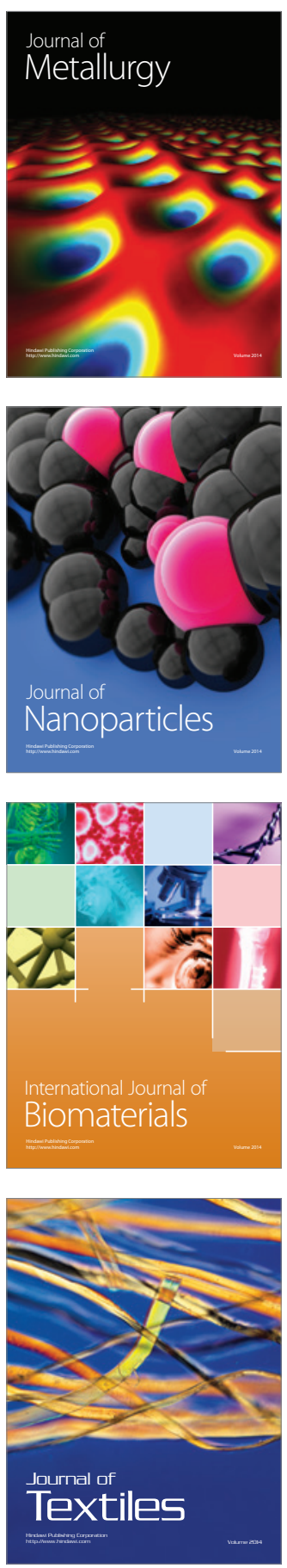